\title{
Transatlantica
}

Revue d'études américaines. American Studies Journal

\section{Le creux et l'excès : jeux d'encre dans The Tunnel de William Gass}

\section{Françoise Sammarcelli}

\section{(2) OpenEdition}

1 Journals

\section{Édition électronique}

URL : http://journals.openedition.org/transatlantica/756

DOI : 10.4000/transatlantica.756

ISSN : $1765-2766$

Éditeur

AFEA

\section{Référence électronique}

Françoise Sammarcelli, « Le creux et l'excès : jeux d'encre dans The Tunnel de William Gass », Transatlantica [En ligne], 1 | 2006, mis en ligne le 06 avril 2006, consulté le 29 avril 2021. URL : http:// journals.openedition.org/transatlantica/756 ; DOI : https://doi.org/10.4000/transatlantica.756

Ce document a été généré automatiquement le 29 avril 2021

\section{(c) (i) (9)}

Transatlantica - Revue d'études américaines est mis à disposition selon les termes de la licence Creative Commons Attribution - Pas d'Utilisation Commerciale - Pas de Modification 4.0 International. 


\title{
Le creux et l'excès : jeux d'encre dans The Tunnel de William Gass
}

\author{
Françoise Sammarcelli
}

1 Je me propose d'explorer ici la façon dont les effets typographiques et les signes visuels sont exploités dans The Tunnel (paru en 1995), roman de plus de 650 pages, fruit d'une lente élaboration (trente ans), dont divers extraits ont d'abord été publiés séparément dans des revues. Dans ce texte-monstre William Gass poursuit sa méditation sur les rapports entre le langage et le monde, et ses expériences avec/sur le lecteur. La masse excessive de ce roman, que Marc Chénetier classe parmi les romans " de la maîtrise " (Au-delà du soupçon, 185), impose au lecteur la conscience de sa propre non-maîtrise : le roman compte trop de pages, trop de signes sur la page, et notre mémoire ne peut prétendre dominer cette somme, ni sans doute prêter attention à chaque détail. De plus on peut commenter l'adéquation entre la forme et la thématique (le «fascisme du cœur »), or ce système s'accompagne d'une occupation très consciente de l'espace de la page : les signes iconiques et les choix typographiques arrêtent le regard, imposent une présence supplémentaire ou excessive par redoublement, par interruption ou défamiliarisation, et confrontent le lecteur au malaise créé par de troubles et dérisoires "matérialités discursives ${ }^{1}$.

2 "My present novel, The Tunnel, is dominated by the trope of its title » expliquait Gass en $1982^{2}$. La métaphore centrale du tunnel introduit en effet tout un réseau métafictionnel du creux et du plein, et les commentateurs n'ont pas manqué de souligner l'homophonie paradoxale du trou et du tout (hole/whole). Claire Maniez commente l'image ambiguë et complexe qui «suggère à la fois la fuite et l'emprisonnement, l'issue et le refuge, le vide et la structure qui l'entoure " (Maniez 105). Le narrateur homodiégétique, William Kohler, historien américain quinquagénaire, auteur d'une somme sur l'Allemagne hitlérienne intitulée Guilt and Innocence in Hitler's Germany, ne parvient pas à rédiger l'introduction de cet ouvrage, et dérape vers des souvenirs autobiographiques entrecoupés de réflexions générales sur l'histoire et le nazisme. Le personnel, l'intime, voire le lyrique se glisse alors dans les creux de l'historiographie. 
3 Simultanément Kohler creuse clandestinement un trou dans le sous-sol de sa maison (« Today I began to dig » 146, voir aussi 501, etc.), puis cache la poussière et la terre dans les tiroirs de sa femme - s'il y a du vide, il y a donc aussi des gravats, du trop. Le tunnel, sous toutes ses formes, est un des éléments importants de la diégèse (par exemple Kohler se retrouve coincé dans une conduite d'entretien), mais il fait l'objet de ce que j'appellerai une sursémantisation: par la voix du narrateur, il est aussi explicitement associé au tunnel chez Lewis Carroll, en fait au terrier (« rabbit-hole »), où le corps d'Alice subit des transformations («Alice in her tunnel could not have improved on the phenomenon ", Gass 27). De surcroît, après avoir évoqué un pasteur particulièrement éloquent entendu dans sa jeunesse, Kohler discute le pouvoir de la parole, des mots, et entre autres la façon dont Hitler en aurait pris conscience : « Adolf Hitler had a similar experience-only it was in that movie about a political agitator he saw in Vienna, the one made from a Kellermann novel. Der Tunnel, it was titled. Yes. At least I've got that right. Though K. may be spelled with one n. I'm not sure" (39) - l'hésitation, à la façon d'une hypallage ou fausse attribution, ne porte pas sur ce qu'on attendrait. Kohler a aussi lu Kafka, mais il s'imagine plus loin, surpris par sa femme à la cave, répondre "I'm thinking of digging a tunnel-you know-to escape from the camp » (148).

4 Effets de surface et de profondeur (collages, caractères gras, surimpressions, relief et béance), le travail polémique entrepris par l'écrivain contribue à faire de la page un champ d'expérimentation, sinon de dégradation, dont j'analyserai quelques enjeux.

Le visible et l'illisible : un texte qui prend forme ou qui fait tache

Dans le même temps qu'il provoque, le texte confronte d'emblée le lecteur à des formes en couleurs sur la page, à la conscience aiguë d'une inscription, et le met au défi de nommer ou décrire (ou de-scribe). Telle stratégie, en soi, n'est pas nouvelle chez Gass. Willie Master's Lonesome Wife (paru en 1968) donnait littéralement corps à l'activité textuelle en utilisant des papiers de couleurs différentes, offrant dès la couverture dédoublée une image du corps nu de l'épouse, en multipliant les renvois, les astérisques, etc, et inspirait à Marc Chénetier le commentaire suivant dans Au-delà du soupçon: «le texte prend corps, se fait corps, le corps se verbalise et se déploie au fil d'un récit-alibi où le thème est strictement forme(s) » (119).

6 À cette stratégie de l'in-formation se rattache notamment, dans The Tunnel, l'insertion de nombreux calligrammes : "The Drunkard's song» (646-648) imite les titubations de l'ivrogne, "what a way to weave ", et sa typographie ondule de gauche à droite sur la page ; ailleurs l'évocation des tornades dans le midwest (112) s'agrémente d'un court « texte » dont la mise en page évoque le mouvement de la masse d'air dans le cyclone :

$$
\begin{gathered}
\text { kyklos } \\
\text { cyclone } \\
\text { tornar } \\
\text { tornado } \\
\text { whirlwind } \\
\text { Wind und Wasserhosen } \\
\text { god's soda straw } \\
\text { hog-swal } \\
\text { l } \\
\text { o } \\
\text { w }
\end{gathered}
$$




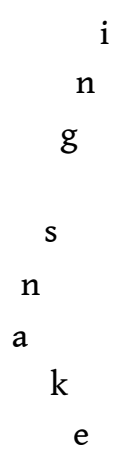

8 Mais ce n'est pas tout, car la tache tend à faire fonctionner l'illisible et l'intransitif (ou $\mathrm{du}$ moins à saturer le code herméneutique), quand elle ne s'ingénie pas à créer des effets de choc en contexte, confrontant le lecteur à une forme de violence qui est la violence de l'imprévisible. Dictature de l'effet ou recherche très aboutie sur les modes de la défamiliarisation? Il semble bien en tout cas que le recours à l'hétérogène, comme le collage de documents - extraits de témoignage, de règlements (26-27, 30, etc.) - batte en brèche l'uniformisation et les idéologies qui la revendiquent. Les cent premières pages du roman, notamment, concentrent un grand nombre d'interventions typographiques - page représentant des cartes de visite sur un plateau (79), extraits de «lettres d'excuses", colonnes, listes, emprunts aux codes de la bande dessinée $(15,2527,43)$ - qui coïncident avec de nombreuses références au nazisme et aux camps d'extermination, comme si jouer avec la forme permettait d'échapper à l'arbitraire oppresseur des organisations convenues, ou d'exorciser une mémoire non réconciliée.

9 Contre les interprétations qui tendent à dédramatiser la crise, je dirai qu'on ne tourne pas la page à la première lecture sans une certaine appréhension, parce qu'on ne sait pas ce qui va suivre. Un numéro de page peut être remplacé par une inscription évoquant un tatouage (00031 en haut de la page 31, 00033 au milieu de la page 33) ; ainsi la page n'est pas seulement une page, elle est aussi un prisonnier dans un camp - pourtant un peu plus loin la page 40 reprendra le procédé, mais pour montrer un billet de concert! Aux pages 48-49 quatre colonnes obsédantes, comme des additions, («shot " «shot » «shot » « hang », complété par « gas ») s'intercalent entre les lignes du discours de Kohler sur l'histoire, semblent brièvement se fondre avec lui «History is just such a sum-the upshots of upshots»), puis par le jeu des interlignes variables, elles le parasitent, le mot "gas " venant se juxtaposer à "remember ", à " amplitude » ou à «water »: la violence de la juxtaposition dés-articule le discours policé de l'historien.

10 Vers la fin du livre, une page (où une longue phrase refuse de nous laisser reprendre haleine) fait ressortir, au milieu d'une liste, le motif de la tache, aussi insignifiante soitelle. De la sorte, l'expression "stain of tea ", en gras, tend à reproduire la valorisation que, selon Kohler, le poème apporte aux objets du quotidien: «The true poem is a community of words formed from the clutter of life, and by virtue of its ceremonies the true poem confers citizenship on all the modest items which cover, for instance, a work-torn desk like mine with their concerns » (642).

11 Le livre est d'abord un objet à voir, ce que les signes iconiques et les effets visuels annoncent dès le paratexte. Si les premiers éléments visuels, les deux fanions en couleurs ("pennants of passive attitudes and emotions »), aussi dérangeants soient-ils, se laissent appréhender tant bien que mal; si, avant la page portant la dédicace, deux 
dessins se prétendent encore figuratifs (une décoration inquiétante, «medal for ingratitude » et au-dessus une bannière «PdP banner »- où PdP, on le découvre plus tard, pourrait être l'abréviation de "Party of the Disappointed People ", (266), ou encore de "Party of the Disappointing Penis ", (384) - tous les dessins ne se laissent pas ainsi «décoder ». Du reste, quand le texte multiplie les offres d'interprétation, comme pour une page de petits dessins et drapeaux (267), il propose soit des indices et légendes redondants ("Fez», "cloth cap», «bars for bars ») soit des marqueurs de subjectivité ("violet for indignation, resentment », "yellow for weakness, cowardice, passivity »), qui dé/recontextualisent les signes d'appartenance.

Le dessin qui figure sur la page 2 ne se prête pas directement à l'identification (ni guère même à la description) : un cercle enserre une forme rouge sur fond blanc, comme un test de Rorschach pour un lecteur en mal d'associations : ainsi on pourrait y voir un semblant de symétrie et deviner un insigne hitlérien (l'aigle) aux contours estompés. Or cette figure réapparaît sous forme dégradée $(57,267,288)$. Ainsi on trouve page 57 une référence à Proust, suivie d'un dessin encore moins figuratif que celui de la page 2 , en violet, rouge et blanc (un cercle contenant une forme non identifiée). Encadrée de noms de quatre mois comme des points cardinaux (aug, july, may, june), cette forme apparait dans un contexte qui associe les thèmes de l'écriture, de l'homosexualité masculine et de certaines pratiques: « 0 sure, we know why Proust wrote: to justify one man's sordid sadomado ways to the interested asses of other men. and that as we also know, requires an endless book ». Le lecteur se dit alors qu'il y a peut-être là le dessin d'un anus, et ce qui passait pour abstrait bascule dans le scatologique. La scatologie à l'œuvre situe alors les fondations du livre au niveau du fondement - voyez, ennemi lecteur, ce que je vous montre, voyez comment je vous confie la tâche de soupçonner la forme. L'entrée en matière, l'entrée dans le tunnel, correspond donc à une entrée (une percée ?) dans la matérialité de la page. D'autres dessins suivront, et le roman s'achève (652) sur un autre dessin, plus petit et en noir et blanc, à quatre lobes, mais apparemment aussi mystérieux que le premier ${ }^{3}$.

Par ailleurs, la matérialité de l'écriture s'exhibe aussi au moment même où elle joue à se dissimuler, quand des mots en surimpression ( « note note note » en gros caractères, 45) ou des grilles ressemblant à celles des mots croisés (627-628) font voir comme la surface (qu'il faut creuser comme Kohler) et le dessous, comme deux épaisseurs de texte. À la page 45 on devine entre autres ceci, dans un passage sur la mémoire où le mot « note » appelle et retarde le regard:

[...] Stones to mark the selves that are no more. Note. Note. Still, there are ghosts in every grave (except the ones the Germans dug, ghettos underground which snuffed out even spirits), and one may suddenly materialize, condense on the side of the sky like moisture on a glass [...] (45).

Ce sont justement les mots "materialize» et "condense» qui se cachent et problématisent la substance. On peut avancer ici l'hypothèse générale que The Tunnel explore les possibilités d'une forme de la forme, d'un signifiant du signifiant ; que, du coup, la diégèse passe à l'arrière-plan, du côté du non-racontable, non-résumable (le corps dans sa crudité, la Shoah), évoluant entre le trop de présence (l'obscène) et le trop d'absence (les juifs exterminés).

Vers la fin du roman, les grilles de mots croisés introduisent la diversion, l'esquive et sa représentation, mais rendent aussi visibles l'effet des grilles de lecture, le noir et le blanc qui laissent voir ou occultent certains éléments. Agé de quinze ans, Kohler va voir 
sa mère, depuis longtemps alcoolique, qui a été internée dans un hôpital psychiatrique. Dans la salle d'attente, il s'occupe avec des mots croisés, passe-temps maternel. Moins que la tristesse, le texte dit alors l'embarras et la honte, et les cases blanches et noires forment comme un écran (protection contre l'affect ou l'indifférence ?). Les définitions insérées dans le texte, auxquelles le jeune homme ne sait pas répondre, procurent une distance, tout comme elles suggèrent l'opacité dérisoire du monde (627-629).

Le livre qui donne ainsi à voir l'épaisseur des signes sur la page, thématise aussi la masse - embonpoint du personnage (213), corps imposant de sa femme - : excès de chair, excès de la chair, débordements textuels. Mais penser la masse, c'est aussi évoquer les masses populaires qui ont soutenu le nazisme. Hannah Arendt, parmi d'autres, a insisté sur le rapport entre les masses et le totalitarisme (pouvoir du nombre et de l'indifférence) : « les mouvements totalitaires visent et réussissent à organiser des masses - non pas des classes [...] les mouvements totalitaires dépendent de la seule force des nombres ", «ils sont possibles partout où se trouvent des masses, qui, pour une raison ou pour une autre, se sont découvert un appétit d'organisation politique " (Le système totalitaire, 29-30, 31-32). Comment glisse-t-on du quantitatif au qualitatif, de l'excès à la dictature ? Mais encore, comment passe-t-on de la masse à la substance ?

L'interrogation sur la substance, sur ce qui serait le cœur du cœur du texte, substitue la guerre des mots aux jeux de mots (24-25). Rythmés par les « rat tat tat » qui rappellent le bruit des mitrailleuses, ils suggèrent que le langage lui-même ne peut pas toujours survivre à l'horreur, ni lui redonner forme :

dry spiteful speech, jailed conjunctions, metaphors machine-gunned where they stood, rat tat tat tat [...] total word war, tat and letters which have thrown away their arms and fled the front, tat not to mention tat those lost in service tat down in the fight, all the lllll's gone into the repeated spelling of 'hell', so we are -eft with -ife and -aw and -ove and -onging, -itt-e more, as if our thought were starting over in its mewling infancy, inside its seed, although the spirit, ah! the spirit, 0 the spirit has stopped. tat. stopped. (24-25)

18 Si les mots manquent, s'ils s'amenuisent sur la page, menacés de disparition, dans l'effrayante supplique où les glissements phonétiques et les signifiants massacrés ne garantissent pas un quelconque sursis - «a morning only > please : a moment more > please : a breath before at last ! the long Last tat or after tat WHAT ? on liptoe WHAT ? at liptip WHAT ? at pitlip 0 in limeslime What? what ? what?» (25) - , la dégradation ou la déchéance touche la substance, impose une autre position, en dessous? souterraine ?: "Sub-stance. Listen to the mind munch. Consonants, General, explode like grenades. Vowels rot in some soft southern mouth, and meaning escapes from those oooos as from an ass » (25).

Peut-on sauver la langue du désastre et quel serait l'enjeu de cette flambée d'autodestruction? S'agit-il alors pour Gass de «resensibiliser la langue, qu'elle palpite plus qu'elle ne raisonne », ce qui fut le projet revendiqué par Céline ${ }^{4}$, cité par Julia Kristeva dans Pouvoirs de l'horreur? Gass joue un jeu curieux et, pour Kohler, écrivain désenchanté, la poésie perdue se convertit avec hargne dans les références obsédantes à tout ce qui sort du corps humain (sécrétions, sperme, urine, matières fécales...).

L'excès et l'obscénité

On l'a compris, Gass ne se contente pas de lancer un pavé dans la mare du " politiquement correct ». La complaisance dans l'obscène, peut-être affichée d'emblée, est revendiquée par la voix narrative nostalgique d'un passé plus heureux, «the good old obscene days» (91). Sur la page suivante le texte-sexe impose au lecteur une 
représentation schématique des organes sexuels masculins quand Kohler raconte «ses femmes ». Il importe de souligner que cette obscénité conjugue explicitement une dimension sexuelle et une dimension raciste: «I think we felt the need to be a little dirty, a little vulgar in our privacy " commente le narrateur (90), mais cela s'entend aussi avec, au sein du couple, un antisémitisme partagé. Le jeune couple Kohler s'excite à coup de plaisanteries grasses (Herr Rickler devient le surnom du pénis) et de jeux de mots épouvantables, voire s'amuse du recyclage parodique de termes porteurs d'une mémoire tragique - seuls les caractères gras signalent une conscience de ce que rien n'est anodin :

I taught Mule [Martha] the difference between Gesamtlösung and Entlösung so well she would propose a total solution to the cockroach or the Kleenex question as readily as I might suggest a suitable solution for the dogs and children of our neighbors, and she has never forgiven me for that contamination. (91)

21 La rancune de l'épouse est-elle partagée par le lecteur ? La stratégie qui consiste à faire surgir l'imprescriptible (les crimes contre l'humanité) à la surface du texte permet-elle de manifester le mépris du lisible?

Multipliant ces jeux de brouillage et de gribouillage, ces recherches sur l'informe et les limites de la représentation où les références à l'Holocauste oscillent entre l'indicible et le banal, à quel moment le texte, au-delà du douteux, tend-il vers l'insupportable ? Ainsi la page 29 donne à voir en creux une étoile à six branches dont les contours sont formés par la liste des prénoms juifs autorisés sous le 3ème Reich, masculins à gauche, féminins à droite. Le motif est précédé et suivi par un extrait du règlement définissant le port de l'étoile, mais il est aussi commenté par un narrateur qui se pique de désinvolture mais s'installe dans l'abjection et l'abomination :

I write these names down slowly, as if I cared, forming the letters with a certain calm disdain. I arrange them emblematically (for am I not at play ?), forming a star my imagination floods with yellow like urine. It gives me pleasure. They are strange names, for the most part; dug out of biblical crannies like tiny obstinate weeds. [...]

Kohler compare cette étoile, cette forme, à son livre sur Hitler, d'une part en ce qu'elle donne en effet à voir et à penser (" exposes itself the way my work exposes the parts and conditions of their crime»), et surtout parce qu'elle impose un ordre, un agencement qui lui dicte ce commentaire réflexif :

my book cries out its commands, and events are disposed like decorative raisins on a cookie. [...] confusion is scarred from the corn, an empty field is ringed with quotes like barbarous wire; well in the same way this pretty pattern of names removes disgust from a dozen dossiers, rips up some threatening proclamations, decorates death like a pennant on a spear. (31)

Opérant la substitution de "barbarous wire " au "barbed wire » attendu, le texte semble émettre un jugement que l'enchaînement de métaphores et de comparaisons induit aussi. Ecrire c'est toujours structurer, donner forme ; c'est selon Derrida affaire de police, mais ici la dimension esthétisante liée au motif de la décoration est particulièrement en cause. L'écriture poétique est-elle encore de mise derrière l'entreprise de dévoration (que suggère l'image du gateau) ? Quant à l'image, "an empty field is ringed with quotes like barbarous wire", elle empoisonne pour longtemps les citations à venir. Que contient le livre?

Le texte explicite la question du malaise et programme notre répugnance au fil de certaines pages où la logorrhée de Kohler nous impose un florilège de pensées totalitaires. Le personnage pose la question du rejet, constate que le haut le cœur n'a 
pas eu lieu: "Is there any way of digesting facts like this-like this one-as Susu digested the handy phallic thumb-sticks of her Jews? » (115). Si le geste abominable de Susu ne l'empêche pas de digérer, le texte s'attaque à la question de l'abjection. Peut-il y avoir de l'abject s'il n'y a pas de reste? Au-delà des tabous (le crime contre l'humanité associé au cannibalisme), la conjonction de l'alimentaire et de la barbarie rejoint la réflexion de Julia Kristeva sur l'abjection. Contre la voix narrative, c'est le visuel qui revient, mais la violence du texte littéraire réside en ceci qu'il fait de la référence à la perversion, à la barbarie littérale, son propre mode de résistance.

Le texte qui creuse l'abject interroge indirectement la question du refoulement. De façon symptomatique, les colères de ce Kohler, auxquelles il consacre de nombreux passages, ont rarement pour objet l'Histoire et ses crimes, mais ses déceptions et déboires personnels, ses rapports avec ses collègues, sa vie conjugale ratée. Cette inversion en elle-même est faite pour déranger, comme les longs passages consacrés à l'historien Magus Tabor. La haine suinte, la querelle protéiforme fait grincer les pages (180 seq), au point qu'on peut se demander pourquoi le fanatisme ou le sectarisme méritent autant le droit à la parole (cf. la section intitulée « being a bigot », 522 seq).

Même si selon Kohler la culpabilité et l'innocence n'ont pas de statut ontologique dans l'histoire, et sont des constructions idéologiques, le thème de la culpabilité est omniprésent, et on pourra de plus lui associer le personnage de Culp, collègue de Kohler, qui écrit un cycle de limericks, au nom de l'obscénité revendiquée :

So the point is, Culp says, the point is to use this very orderly little object [...] to manufacture disorder and to confound whatever it contains. The limerick is an instrument of disrespect. It is a tune toilet, a toy box. It gossips, it slanders, it ruins, it sneers, it snobs. It is a turd tub, a cock lock. (165)

On sait que la place excentrique du limerick dans la poésie anglaise tient à ce que c'est une courte pièce en vers d'un comique absurde et fréquemment grivois. Gass trangresse cette tradition de légèreté et radicalise l'absurde. Par là-même le statut d'une forme poétique est remis en cause. Je reprends volontairement un exemple choisi par Jean-François Chassay dans son essai «Creuser l'histoire: The Tunnel de William Gass » (222) :

A nun went to bed with Herr Hitler,

whose cock just got littler and littler.

$O$ what I would do

If you were a Jew,

he cried as he bit her and hit her. (Gass, 175)

L'hybridité, quand elle intervient, introduit une tension supplémentaire. Un exemplelimite est celui du passage par les formes de la culture populaire, les « funnies » (cf. 15, 26, 208): leurs onomatopées, leur graphisme bien net, détonnent sur fond d'holocauste - et l'on sait que la bande dessinée Maus de Carl Spiegel, fils d'un survivant de Buchenwald, déjouait la question de la représentation impossible en figurant les humains par des animaux : des souris et des chats notamment. Chez Gass, la stylisation graphique, empruntée à la bande dessinée, fait ressortir le dérisoire (bruits de pets !), mais le texte raconte trop les totalisations pour autoriser constamment une lecture en termes de carnavalisation. Inversement l'inclusion de poèmes dans la prose narrative se heurte à notre méfiance (on s'attend toujours à un limerick...)

La question centrale concernant ces effets typographiques pourrait s'énoncer ainsi : les jeux d'encre, explorant les limites du paratexte et les aléas du visible, relèvent d'une mise en tension des rapports d'insertion/isolement, inclusion/exclusion; on peut donc 
considérer qu'ils font recirculer dans la forme de la page la question de l'itératif et du singulatif, de la réduction du multiple au même dont la diégèse semble abuser (cf. « the Jew ", "Jews", "Those Hebrews taught me how to be a bigot ", 91). La typographie, dans l'écart entre des pages compactes sans alinéa et des pages saturées d'artifices, redouble-t-elle les horreurs de la diégèse ou les dément-elle ? Les effets de distinction (arbitraire ou motivée) ou d'indistinction revêtent-ils une valeur pédagogique ou induisent-ils perversement une participation du lecteur à une vaste entreprise de relativisation? C'est dans ce rapport du même à l'autre que se joue notre aventure dans le souterrain de Kohler. Rien ne nous interdit de penser que l'instabilité du procédé évite la fermeture par le changement d'échelle.

Vers quelle extériorité : l'impossible dehors?

31 "What is a book but a container of consciousness, a draft of cantos? » (69). Si l'on entre dans ce roman comme l'on pénètrerait dans un tunnel, cela signifie-t-il qu'on en reste prisonnier? "You shall listen. I shall tell you, » assène le professeur Tabor, surnommé Mad Meg (270). Dans quel état pourra-t-on en sortir ? Le tunnel en effet évoque aussi le conduit auditif et le roman s'achève sur la perspective d'un suicide : « Revolver like the Führer near an ear. Or lay my mind down by sorrow's side » (652). Comme le soulignait Gass, réagissant à une comparaison avec Kafka : «Now Kafka's burrows aren't tunnels exactly, because a tunnel is presumably going from A to B, in order to get through barriers, to get beyond obstacles, to escape. But of course, in my book, the tunnel is itself the point, you're not going anywhere »(Saltzman, 130).

On peut aussi se demander si, dans certains cas, les excentricités typographiques, quand elles redoublent la diégèse, prolongent l'effet d'enfermement et de claustrophobie. Ainsi la section " Mad Meg in the Maelstrom " présente page 8 sur neuf lignes un paragraphe comme évidé en son centre, le texte étant aligné de part et d'autre d'un blanc (à remplir par l'imaginaire) qui figure la fenêtre de solitude ou de non-responsabilité désirée par le personnage: «on this windless page, where I'm beseeched by no one, heard by no one-unaffected, unaffecting-and can point my own direction if I any longer have one ; live as though I have (and had) a life; let this vacant paper window frame a world ". L'image de la fenêtre comme signe d'un éternel dedans, ou d'un désir de sortir...

Plus loin (174), Gass s'offre une page grise avec des zones plus ombrées, entraves à la lecture, imitant des effets de pliure ou de taches, et portant en majuscules l'inscription: «NEW DOUBLE STRONG / EXTRA HEAVY BAG/ NO DOUBLE BAGGING NEEDED » en écho à la page marbrée de Tristram Shandy. Or non seulement le texte, évoquant la rupture avec Lou, la femme aimée, décrit un sac en papier contenant son déjeuner, mais le narrateur rumine son malheur en des termes qui déplient le chagrin sous le jeu de mot : «God, what an expression! what an idea! I've been given the sack. I'll take any opening offered. The surface of you was all the deep I needed. And you felt surface too when my tongue went dancing [...] Lou must have wanted to give me the sack for some time » (175). Gass se moque mais travaille le retour du littéral.

La relation entre le dehors et le dedans tient aussi à l'ambiguïté du paratexte. Dans The Tunnel les effets typographiques contribuent à un jeu de confusion entre texte et paratexte, et redynamisent la notion de seuil ou de frontière comme la notion d'appartenance, en rapport avec un texte qui est entre-dit et qui se cache ( I'll want a private page to hide between each public page of $G$ \& I to serve as their insides, not the tip but the interior of the iceberg ", 92). Dans cet énorme roman, il semble notamment 
que l'emploi qui est fait des intertitres problématise la notion de division. En particulier, l'incertitude sur le niveau est suscitée par la présence de divers énoncés ou expressions distingués par la typographie. Qu'est-ce qui fait passage, qu'est-ce qui fait titre? Si l'on adopte le même critère que Claire Maniez (les expressions en grosses capitales) on peut dénombrer treize sections de «Life in a Chair » à "Outcast in the Mountains of the Heart ». Reste pourtant que quand un mot ou groupe de mots revient avec des variantes typographiques (corps plus grand, emploi des gras mais pas des capitales par exemple), on hésite sur son niveau et, inversement, dans plusieurs cas, les effets de différenciation et mise en relief typographique pourraient fugitivement faire passer pour un intertitre telle ou telle expression (par ex: $\mathrm{N}^{\circ} 2$ Whitefish, 59, ou bien "Confession» et «The Complete Dishonest and Unwholesome Truth», 21). Dans tous ces cas le texte brouille les repères, en donne trop ou pas assez, et le jeu participe d'une déhiérarchisation du sens. De plus quand un titre est répété («Outcast in the Mountains of the Heart », 583 et 632), quand la même expression fonctionne comme titre ou sous-titre et revient avec insistance ("Mad Meg », 214, répété (236) avec une typographie différente, puis $244,245,247)$, il ne dit pas seulement l'obsession ou le radotage de Kohler, il met en faillite le désir de distinction - voire suggère qu'au lieu d'avancer dans le tunnel, le lecteur (ou le récit) fait du sur-place, que le texte ne mène nulle part sauf à penser des échappées dans les profondeurs (au cœur du cœur des ténèbres ?)

35 A cette dynamique se rattache, à mon sens, une double exploration, celle du très (trop ?) explicite dans la dénotation et celle de la saturation de connotations, qui, par contamination, en contexte, peut faire du gaz, d'un nez, d'une barbe des rappels fracassants du racisme. Si l'horreur peut se cacher dans le moindre mot, si l'indicible résonne encore et toujours dans le banal, il n'y a peut-être plus d'extériorité, plus de salut. Le livre alors fait de tous ses lecteurs des coupables en puissance.

Quel est l'interdit qui risque de peser sur la citation? Quelle est donc la responsabilité du lecteur? Le narrateur de The Tunnel s'interroge pour sa part sur les limites de l'investigation des profondeurs:

We sail the seas and fly the skies and drive up and down all the road, but the deepest caves, the cleverest caverns, cannot take us to the underground, tell us what goes on in that inner realm, however it happens [...] whether, at the genetic center of the self, in pure birth earth, we're in that fabled place where compacts of conclusion coalesce like veins of coal, compressed past the thought of further futures and consequently beyond each form of the past, to be free of time like the proverbial bird, fixed-[...] that's what we really don't know and maybe motivates my burrowing-if there's a bottom nature, and just what's what where the well ends, when we pass beneath its water, when we actually enter 'in' and find ourselves in front of $\mathrm{n}$ and on the other side of $\mathrm{i}$. (501)

Le texte semble ainsi creuser un dedans de la forme. La violence du langage, si on la définit du côté du reste, s'acharne contre la perte. Mais si l'on pense aussi l'expérience typographique comme une figure de l'altération, c'est-à-dire d'un devenir autre de la forme, alors on n'est pas loin de la réflexion menée par Laurent Jenny sur le figural : la représentation est susceptible de devenir présentation ${ }^{5}$. Le lecteur réticent peut refermer le livre avant la déflagration finale, mais il ne peut pas esquiver le pouvoir performatif de ces troublants jeux d'encre. 


\section{BIBLIOGRAPHIE}

Arendt, Hannah. Les Origines du totalitarisme : le système totalitaire. Trad. fr. J.-L. Bourget, Robert Davreu \& Patrick Lévy. Paris : Seuil, 1972.

Bakhtine, Mikhaill. Esthétique et théorie du roman. Trad. fr. Daria Olivier. Paris : Gallimard NRF, 1978.

Chassay, Jean-François. Fils, lignes, réseaux. Essai sur la littéature américaine. Montréal : Liber, 1999.

Chénetier, Marc. Au-delà du soupçon : la nouvelle fiction américaine de 1960 à nos jours. Paris : Seuil, 1989.

Gass, William H. «Tropes of the text ", Representation and Performance in Postmodern Fiction. Ed. M. Couturier, Delta, PU de Montpellier, 1983. pp. 33-47.

Gass, William H. The Tunnel. Normal, IL : Dalkey Archive Press, 1995.

Jenny, Laurent. La Parole singulière. Paris : Belin, 1990.

Kristeva, Julia. Pouvoirs de l'horreur : essai sur l'abjection. Paris : Seuil, 1980.

Maniez, Claire. William H. Gass : l'ordre de la voix. Paris : Belin, coll. Voix américaines, 1996.

Saltzman, Arthur M. « An Interview with William Gass ». Contemporary Literature XXV : 2 (Summer 1984).

\section{NOTES}

1. Je détourne ici la formule de Michel Pécheux, spécialiste de l'analyse du discours. Cf. l'ouvrage collectif Matérialités discursives, (PU de Lille, 1981) qui regroupe des textes de Bernard Conein, Jean-Jacques Courtine, Françoise Gadet, Jean-Marie Marandin et Michel Pécheux.

2. "Tropes of the text ", in Representation and Performance in Postmodern Fiction. Ed. M. Couturier, 46.

3. Signalons toutefois que, selon Gass lui-même, ce dessin serait (aussi ?) la coupe d'un rectum. Je remercie Claire Maniez de m'avoir signalé l'interview diffusée sur Internet d'où sont tirées les remarques suivantes :

"Of course, a rare book is nice to have, but what I have is a working library. That's incredibly important to me. I like being surrounded by books. After many years of accumulating books, I can go to my own library and choose the books I need. Oddly, I don't use a library very much any more because I have my own. We spend a lot of our income on books. Sometimes they come in by crates, a hundred at a time. They are all bought on speculation, in a sense, because we don't know when we will use them. I picked up a book once on histology. It had all these anatomical drawings in it, and it sat around for twenty years. I never used it until I wanted a certain design for The Tunnel, and there it was, a cross section of the rectum. I put it at the end of the book. I just never know when I am going to want these things or how odd it will be when I do. I'll keep any book, even if it's a chemistry textbook. » (The Magazine, Author Interviews, «From Experience to Innocence : An Interview with William H. Gass », Jennifer Levasseur and Kevin Rabalais, Vol. XXIII : 1 (Winter 2001). 
4. Lettre à Hindus, 15 mai 1947, repr. L'Herne, 113, cité dans Pouvoirs de l'horreur, 225.

5. Voir le premier chapitre de son livre La Parole singulière, et entre autres cette définition : «Le figural est donc doublement « représentatif ». Il représente (imitativement) quelque chose du monde en re-présentant (en présentant à neuf) la forme de la langue. » (26)

\section{AUTEUR}

\section{FRANÇOISE SAMMARCELLI}

Paris 4 